\title{
Optimal persistent disturbance attenuation control for linear hybrid systems
}

\author{
Hai Lin ${ }^{\mathrm{a}}$, Guisheng Zhai ${ }^{\mathrm{b}}$, Panos J. Antsaklis ${ }^{\mathrm{c}, *}$ \\ ${ }^{a}$ Department of Electrical and Computer Engineering, National University of Singapore, Singapore 117576, Singapore \\ ${ }^{b}$ Department of Mechanical Engineering, Osaka Prefecture University, 1-I Gakuen-Cho, Sakai. \\ Osaka 599-8531, Japan \\ ${ }^{c}$ Department of Electrical Engineering, University of Notre Dame, Notre Dame, IN 46556, USA
}

\begin{abstract}
In this paper, the disturbance attenuation properties for a class of linear hybrid systems are investigated, and a hybrid optimal persistent disturbance attenuation control problem is studied. First, a procedure is developed to determine the minimal $l^{\infty}$ induced gain of linear hybrid systems. However, for general hybrid systems, the termination of the procedure is not guaranteed. Then, the decidability issues are discussed. The termination of the procedure in a finite number of steps is shown for a subclass of hybrid systems with simplified discrete event dynamics, called switched linear systems. Finally, the optimal persistent disturbance attenuation controller synthesis problem is studied. It is shown that the optimal performance level can be achieved by a piecewise linear state feedback control law, and a systematic approach is proposed to design such feedback control.

(C) 2005 Elsevier Ltd. All rights reserved.
\end{abstract}

Keywords: Disturbance attenuation; Robust performance; Hybrid dynamical systems; Switched systems; Invariant sets; Induced gain

\section{Introduction}

Hybrid systems are heterogeneous dynamical systems, the behaviors of which are determined by interacting continuous variable and discrete event dynamics. By heterogeneity, we mean

\footnotetext{
* Corresponding author. Tel.: +1 (574) 631 5792; fax: +1 (574) 6314393.

E-mail addresses: hlin28@gmail.com (H. Lin), zhai@me.osakafu-u.ac.jp (G. Zhai), antsaklis.1@nd.edu (P.J. Antsaklis).
}

$0362-546 \mathrm{X} / \$$ - see front matter (C) 2005 Elsevier Ltd. All rights reserved.

doi:10.1016/j.na.2005.12.014 
hybrid systems containing two different kinds of dynamics. One is time-driven continuous variable dynamics, which is usually described as differential or difference equations; the other is event-driven discrete logic dynamics, whose evolution depend on if-then-else types of rules. In addition, these two kinds of dynamics interact with each other and generate complex dynamical behaviors, such as switching once a certain continuous variable passes through a threshold, or state jumping upon certain discrete event occurring etc. Hybrid systems have been identified in a wide variety of applications in control of mechanical systems, process control, automotive industry, power systems, aircraft and traffic control, among many other fields.

The history of hybrid system research can be traced back at least to the 1950's with the study of engineering systems that contain relays and/or hysteresis. However, hybrid systems began to attract people's attention in the early 1990's, mainly because of the vast development and implementation of digital micro controllers and embedded devices. The last decade has seen considerable research activities in the field of hybrid systems involving researchers from a number of traditionally distinct fields, such as computer science, control systems engineering, and mathematics $[1,2]$. The issues studied include modeling [3-6], reachability analysis $[4,7,8]$, stability and stabilization [9-14], observability and controllability [15,3,16] and optimal control $[17,18]$ primarily. However, the literature on robust control of hybrid systems is relatively sparse.

In this paper, we will focus on the induced gain analysis and robust optimal control for classes of linear hybrid/switched systems. The robustness here is with respect to the exterior disturbances. There are some related works in the literature on analyzing the induced gain in switched systems. In [19], the $\mathcal{L}_{2}$ gain of continuous-time switched linear systems was studied based on piecewise quadratic Lyapunov functions incorporated with an average dwell time concept, and the results were extended to the discrete-time case in [20]. In [21], the rootmean-square (RMS) gain of a continuous-time switched linear system with slow switching was computed in terms of the solutions to a collection of Riccati equations. There are also papers on the robust controller synthesis for hybrid systems, see for example [22,23]. In [22], a multiple output feedback controller framework was employed to solve $\mathcal{H}_{\infty}$ control problem of a LTI plant. The controller design was reduced to solutions to a dynamic programming equation and a Riccati equation. The $\mathcal{H}_{\infty}$ control problem for piecewise affine systems was investigated in [23] based on dissipativity theory and LMI techniques. These robust performance and control problems considered in the literature so far are all in the signal's energy sense, and assume that the disturbances are constrained to have finite energy, i.e., bounded $\mathcal{L}_{2}$ norm. In practice, there are disturbances that do not satisfy this condition and act more or less continuously over time. Such disturbances are called persistent [24], and cannot be treated in the above framework. In this paper we consider $l^{\infty}$ induced gains to deal with the robust performance problems in the signal's magnitude sense, i.e., time domain specifications.

The persistent disturbance attenuation properties for hybrid/switched systems have been considered in our previous work [25-27]. In [26], a class of uncertain switched linear systems affected by both parameter variations and exterior disturbances was considered, and the uniformly ultimate boundedness control problem was studied for both discrete-time and continuous-time cases. Under the assumption that each subsystem admits a finite persistent disturbance attenuation level, it was shown in [26] that, by proper switching, the closed-loop switched systems could reach a better disturbance attenuation level than any single subsystem. An optimal disturbance attenuation property for uncertain switched systems and its decidability issue were discussed in [25]. The results for optimal disturbance attenuation property analysis were extended to classes of general uncertain hybrid systems in [27]. All of these previous works are analysis results on the disturbance attenuation property without addressing the controller 
design. This paper is an extension of [27] and addresses the robust optimal hybrid controller synthesis problem.

This paper is organized as follows. In Section 2, we first define the linear hybrid systems with persistent external disturbances. Then, the $l^{\infty}$ induced gain analysis problem for the linear hybrid system and its robust optimal controller synthesis problem are formulated. After introducing some necessary preliminary results in Section 3 , the $l^{\infty}$ induced gain analysis problem is investigated in Section 4, and a bisection-based procedure is proposed to determine a nonconservative bound on the optimal disturbance attenuation level. The decidability issues of the proposed procedure are discussed in Section 5, and a decidable subclass of the linear hybrid systems, called switched linear systems, is specified. The robust optimal controller synthesis problem is studied in Section 6. A systematic approach to design an explicit hybrid state feedback control law is proposed, which is based on polyhedral algebra and linear programming techniques. Finally, a numerical example is given and concluding remarks are made.

Notation. The letters $\mathcal{E}, \mathcal{P}, \mathcal{S} \cdots$ denote sets, $\partial \mathcal{P}$ the boundary of set $\mathcal{P}$, and int $\{\mathcal{P}\}$ its interior. A polytope (bounded polyhedral set) $\mathcal{P}$ will be presented either by a set of linear inequalities $\mathcal{P}=\left\{x: F_{i} x \leq g_{i}, i=1, \ldots, s\right\}$, and compactly by $\mathcal{P}=\{x: F x \leq g\}$, or by the dual representation in terms of the convex hull of its vertex set $\left\{x_{j}\right\}$, denoted by Conv $\left\{x_{j}\right\}$. For $x \in \mathbb{R}^{\prime \prime}$, the $l^{1}$ and $l^{\infty}$ norms are defined as $\|x\|_{1}=\sum_{i=1}^{n}\left|x_{i}\right|$ and $\|x\|_{\infty}=\max _{i}\left|x_{i}\right|$ respectively. $l^{\infty}$ denotes the space of bounded vector sequences $h=\left\{h(k) \in \mathbb{R}^{\prime \prime}\right\}$ equipped with the norm $\|h\|_{l^{\infty}}=\sup _{i}\left\|h_{i}(k)\right\|_{\infty}<\infty$, where $\left\|h_{i}(k)\right\|_{\infty}=\sup _{k \geq 0}\left|h_{i}(k)\right|$.

\section{Problem formulation}

We consider discrete-time piecewise linear hybrid systems of the form

$$
x(t+1)=A_{q} x(t)+B_{q} u(t)+E d(t), \quad t \in \mathbb{Z}^{+}, \quad \text { if } x \in \mathcal{P}_{q}
$$

where $x(t) \in \mathbb{R}^{n}$ is the state variable, $u(t) \in \mathcal{U}_{q} \subset \mathbb{R}^{m}$ is the control input, and the disturbance input $d(t)$ is contained in $\mathcal{D} \subset \mathbb{R}^{r}$, the $l^{\infty}$ unit ball, i.e., $\mathcal{D}=\left\{d:\|d\|_{l^{\infty}} \leq 1\right\}$. It is assumed that $\mathcal{U}_{q}$ is a polytope assigned to each mode $q$. Let the finite set $Q$ stand for the collection of discrete modes $q$. The partition of the state space $\mathcal{X} \subseteq \mathbb{R}^{n}$ is given as a finite set of polyhedra $\left\{\mathcal{P}_{q}: q \in Q\right\}$, where $\mathcal{P}_{q} \subseteq \mathcal{X}$ and $\bigcup_{q \in Q} \mathcal{P}_{q}=\mathcal{X}$.

Associated with the piecewise linear hybrid system (1), an output $z(t)$ is considered as

$$
z(t)=C x(t)
$$

where $C \in \mathbb{R}^{p \times n}$ and $z(t) \in \mathbb{R}^{p}$. It is assumed that $\left(A_{q}, E\right)$ is controllable and $\left(A_{q}, C\right)$ observable for all $q$.

Our primary concern here is what is the effect of the persistence disturbance $d(t)$ on the output $z(t)$. It is often desirable to make the magnitude of $z(t)$ as small as possible, for example when the $z(t)$ reflects some errors that need to be eliminated. In this paper, we will study such disturbance attenuation effects for the linear hybrid systems introduced above, and aim to design hybrid control laws so as to keep $z(t)$ as close to the origin as possible in the face of unknown disturbances. The control law refers to a map $c: \mathcal{X} \times \mathbb{Z}^{+} \rightarrow Q \times \cup_{q \in Q} \mathcal{U}_{q}$. The control law $c$ is hybrid in the sense that it includes both a rule for active discrete mode selection and continuous variable control signal determination. A hybrid control law $c$ is called admissible, if at every time instant $t$, the discrete mode $q(x, t)$ being selected is a feasible mode for state $x(t)$, i.e., $x(t) \in \mathcal{P}_{q(x, t)}$, and the continuous control signal being determined satisfies $u(x, t) \in \mathcal{U}_{q(x, t)}$. 
For this linear hybrid systems (1) and (2), we are interested in determining a non-conservative bound for the $l^{\infty}$ induced norm from $d(t)$ to $z(t)$ that can be achieved, which is defined as

$$
\mu_{\mathrm{inf}}=\inf \left\{\mu \mid \exists x_{0},(q(t), u(t)) \in Q^{*} \times \mathcal{U}^{*}:\|z(t)\|_{l \infty} \leq \mu, \forall\|d(t)\|_{l^{\infty}} \leq 1\right\}
$$

where $Q^{*} \times \mathcal{U}^{*}$ stands for the set of all the sequences of $q(t)$ and $u(t)$ that are admissible. If the undisturbed linear hybrid systems, i.e., $\mathcal{D}=\{0\}$, are not asymptotically stabilizable, then there is no finite disturbance attenuation level. Hence, it is necessary to require that there exist admissible control laws asymptotically stabilizing the undisturbed linear hybrid system. In particular, we are concerned about semi-global asymptotic stability, which means that for any finite bound on the initial conditions $x_{0}$ given, there exist hybrid control laws to drive all the initial conditions to the origin asymptotically.

In this paper, we will study the following two problems.

Problem 1 (Robust Performance Analysis Problem). Given the piecewise linear hybrid systems (1) and (2), determine the minimal $l^{\infty}$ induced gain from $d(t)$ to $z(t)$ that can be achieved by some admissible control law.

The second problem is to construct such an admissible control law that guarantees the $l^{\infty}$ induced gain from $d(t)$ to $z(t)$.

Problem 2 (Robust Optimal Control Problem). Given linear hybrid systems (1) and (2), construct an admissible control law, such that the minimal $l^{\infty}$ induced gain from $d(t)$ to $z(t)$, $\mu_{\text {inf, is achieved. }}$

The robust optimal controller synthesis problem is studied in Section 6. It is interesting to notice that the optimal performance level can be achieved by a piecewise linear state feedback control law.

\section{Preliminary results}

The basic idea employed in this paper is to translate the required level of performance into constraints on the controlled system. Then, these constraints can be dealt with by invariant set theory, since state and control constraints can be satisfied if and only if the initial state belongs to some proper invariant set for the closed-loop system. Therefore, we introduce the controlled robust invariant set for the hybrid systems as follows.

Definition 3. The set $\Omega \subset \mathcal{X}$ is controlled robust invariant for the linear hybrid systems (1) and (2) if for all the initial condition $x_{0} \in \Omega$, there exist admissible control laws, such that $x(t) \in \Omega$, $\forall t \geq 0$, despite disturbances.

Invariant set theory has been studied in the literature for decades, see for example [28-31] and references therein. Blanchini gave a compressive review of the invariant set theory in [32]. In [30], a discrete-time linear system with polyhedral state, control and disturbance constraints was considered, and the controlled invariant set was geometrically and analytically characterized. The authors of Ref. [33] considered a class of discrete-time hybrid systems with piecewise linear time-invariant flow function and polyhedral constraints. They also discussed two special classes which made the computation of controlled invariant set decidable. The invariant sets for piecewise affine systems have also been studied in [34] based on convex optimization techniques and linear matrix inequalities. In the literature of hybrid systems, a similar concept, 
maximal safety set, has been studied, for example, in [7,35-37]. In addition, controller design and verification based on invariant sets have been studied in [36,38-41].

In this paper, the invariance checking and calculation for $\Omega$ is based on the backward reachability analysis and robust predecessor operator, which will be defined below.

Definition 4. The robust one-step predecessor set, $\operatorname{pre}(\Omega)$, is the set of states in $\mathcal{X}$, for which there exist feasible modes and admissible control inputs to drive these states into $\Omega$ in one step, despite disturbances, i.e.,

$$
\begin{aligned}
\operatorname{pre}(\Omega)= & \left\{x(t) \in \mathcal{X} \mid \exists q \in Q, u(t) \in \mathcal{U}_{q}: x(t) \in \mathcal{P}_{q},\right. \\
& \left.A_{q} x(t)+B_{q} u(t)+E d(t) \in \Omega, \forall d(t) \in \mathcal{D}\right\} .
\end{aligned}
$$

We can also define the one-step predecessor set under the $q$-th mode, $\operatorname{pre}_{q}(\Omega)$, as the set of all states $x \in \mathcal{P}_{q}$, for which an admissible control input $u \in \mathcal{U}_{q}$ exists and guarantees that the system will be driven to $\Omega$ by the transformation $A_{q} x+B_{q} u+E d$ for all allowable disturbances.

Proposition 5. The robust one-step predecessor set $\operatorname{pre}(\Omega)$ for piecewise linear hybrid systems can be computed as follows:

$$
\operatorname{pre}(\Omega)=\bigcup_{q \in Q} \operatorname{pre}_{q}(\Omega)
$$

Therefore, we only need to calculate the one-step predecessor set for each $q$-th subsystem. Proof is omitted here due to space limits, see [42] for details. Notice that the predecessor set of a piecewise linear set $\Omega$ under a single mode, i.e., pre $_{q}(\Omega)$, has been studied extensively in the literature and can be computed by Fourier-Motzkin elimination [43] and linear programming techniques, see for example [29,31] and references therein. Notice that the difficulty in calculating pre $_{q}(\Omega)$ comes mainly from the fact that the region $\Omega$ is typically non-convex. Even if one starts with convex sets, the procedure deduces non-convex sets for hybrid systems after a onestep predecessor operation. Because of the non-convexity, some of the linearity and convexity arguments do not hold and extra care should be taken.

Proposition 6. The robust one-step predecessor set for a (non-convex) piecewise linear set $\Omega$, pre $(\Omega)$, can be written as a finite union of polyhedra.

Although the convexity is not preserved under the one-step predecessor operation, the piecewise linearity remains unchanged as Proposition 6 implies. Therefore, one can apply the predecessor operation recursively, which will be explored in the next section.

\section{Hybrid robust performance analysis}

In this section, we will focus on the first problem and determine the minimal $l^{\infty}$ induced gain from $d(t)$ to $z(t)$ that can be achieved by some admissible control laws for the closed-loop linear hybrid systems. For such purposes, we first introduce the performance level $\mu$ set as

$$
\Omega_{\mu}=\left\{x:\|C x\|_{\infty} \leq \mu\right\}=\left\{x:\left[\begin{array}{c}
C \\
-C
\end{array}\right] x \leq\left[\begin{array}{l}
\bar{\mu} \\
\bar{\mu}
\end{array}\right]\right\}
$$

where $\bar{\mu}$ stands for a column vector with $\mu$ as its elements. Note that $\Omega_{\mu}$ is a polytope containing the origin in its interior. 


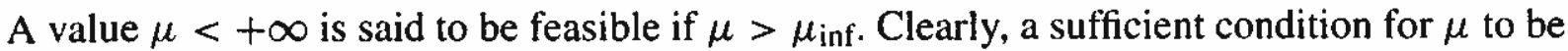
feasible is that the hybrid performance level set $\Omega_{\mu}$ is controlled robust invariant. Therefore, the $l^{\infty}$ induced gain analysis problem is transformed into checking the controlled robust invariance of the disturbance attenuation performance level set. The following is an important, well-known geometric condition [32] for a set to be controlled invariant.

Theorem 7. The set $\Omega_{\mu}$ is a controlled robust invariant set if and only if $\Omega_{\mu} \subseteq \operatorname{pre}\left(\Omega_{\mu}\right)$.

In general, a given set $\Omega_{\mu}$ is not controlled robust invariant. However, $\Omega_{\mu}$ may contain controlled robust invariant subsets. In other words, the sufficient condition for $\mu$ to be feasible may be too conservative. In order to get necessary and sufficient conditions for the admissibility of $\mu$, we first introduce the following definition.

Definition 8. The set $\mathcal{C}_{\infty}\left(\Omega_{\mu}\right)$ is the maximal controlled robust invariant set contained in $\Omega_{\mu}$ for the linear hybrid systems (1) and (2) if $\mathcal{C}_{\infty}\left(\Omega_{\mu}\right)$ is controlled robust invariant and contains all the controlled robust invariant sets contained in $\Omega_{\mu}$.

The uniqueness of the maximal controlled robust invariant set $\mathcal{C}_{\infty}\left(\Omega_{\mu}\right)$, if non-empty, follows immediately from the fact that the union of two controlled robust invariant sets is still controlled robust invariant, and that $\mathcal{C}_{\infty}\left(\Omega_{\mu}\right)$ is a subset of $\Omega_{\mu}$. In order to calculate the maximal controlled robust invariant set in $\Omega_{\mu}$, we introduce the one-step controllable set of $\Omega_{\mu}$ as

$$
\mathcal{C}_{1}\left(\Omega_{\mu}\right)=\operatorname{pre}\left(\Omega_{\mu}\right) \cap \Omega_{\mu} .
$$

It follows from Proposition 6 that the one-step controllable set $\mathcal{C}_{1}\left(\Omega_{\mu}\right)$ is a piecewise linear set if $\Omega_{\mu}$ is given as a piecewise linear set. Therefore, the one-step controllable set operator can be used recursively to define a $i$-step controllable set $\mathcal{C}_{i}\left(\Omega_{\mu}\right)$ as follows.

$$
\mathcal{C}_{i}\left(\Omega_{\mu}\right)=\mathcal{C}_{1}\left(\mathcal{C}_{i-1}\left(\Omega_{\mu}\right)\right)=\operatorname{pre}\left(\mathcal{C}_{i-1}\left(\Omega_{\mu}\right)\right) \cap \mathcal{C}_{i-1}\left(\Omega_{\mu}\right),
$$

for $i \geq 2$. The sequence of finite-step controllable sets $\mathcal{C}_{i}\left(\Omega_{\mu}\right)$ has the following property.

Proposition 9. The sequence of finite step controllable sets $\mathcal{C}_{i}\left(\Omega_{\mu}\right)$ is decreasing in the sense of

$$
\mathcal{C}_{i}\left(\Omega_{\mu}\right) \subseteq \mathcal{C}_{i-1}\left(\Omega_{\mu}\right)
$$

for $i \geq 1$ and $\mathcal{C}_{0}\left(\Omega_{\mu}\right)=\Omega_{\mu}$. The maximal controlled invariant set in $\Omega_{\mu}$ for the piecewise linear hybrid system (1) is given by

$$
\mathcal{C}_{\infty}\left(\Omega_{\mu}\right)=\bigcap_{i=0}^{\infty} \mathcal{C}_{i}\left(\Omega_{\mu}\right)
$$

The proof of this result is similar to the proof of Theorem 3.1 in [29], and it is omitted here.

Based on the maximal controlled robust invariant set $\mathcal{C}_{\infty}\left(\Omega_{\mu}\right)$, we state now the basic result of this section which will be used to give a solution to the disturbance attenuation property analysis problem.

Proposition 10. A value $\mu(<+\infty)$ is feasible, i.e., $\mu>\mu_{\mathrm{inf}}$, if and only if the maximal controlled robust invariant subset of $\Omega_{\mu}, \mathcal{C}_{\infty}\left(\Omega_{\mu}\right)$, is non-empty.

This result suggests the following constructive procedure for finding a robust performance bound.

Procedure 1. Checking whether $\mu>\mu_{\mathrm{inf}}$ 
(1) Initialization: Set $i=0$ and set $\mathcal{C}_{0}=\Omega_{\mu \mu}$.

(2) Compute the set $\mathcal{C}_{i+1}\left(\Omega_{\mu}\right)=\operatorname{pre}\left(\mathcal{C}_{i}\left(\Omega_{\mu}\right)\right) \cap \mathcal{C}_{i}\left(\Omega_{\mu}\right)$.

(3) If $0 \notin \mathcal{C}_{i+1}$ then stop, and the procedure has failed. Thus, the output does not robustly meet the performance level $\mu$.

(4) If the $\mathcal{C}_{i}\left(\Omega_{\mu}\right)=\mathcal{C}_{i-1}\left(\Omega_{\mu}\right)$, then stop, and set $\mathcal{C}_{\infty}\left(\Omega_{\mu}\right)=\mathcal{C}_{i}\left(\Omega_{\mu}\right)$.

(5) Set $i=i+1$ and go to step (2).

This procedure can then be used together with a bisection method on $\mu$ to approximate the optimal value $\mu_{\text {inf }}$ arbitrarily close, which solves the disturbance attenuation property analysis problem. If the procedure stops at step (3), we conclude that $\mu<\mu_{\text {inf }}$ and we can increase the value of the output bound $\mu$. This comes from the fact that if $\mathcal{C}_{\infty}\left(\Omega_{\mu}\right) \neq \emptyset$ then $0 \in \mathcal{C}_{i+1}$. Else, if the procedure stops at step (4), we have determined a feasible bound for the output, say $\mu>\mu_{\text {inf }}$, that can be decreased. The above discussion can be formalized as the following bisection algorithm:

Algorithm 1. Algorithm for calculating $\mu_{\text {inf }}$

(1) Initialization: Choose the initial interval $\left[\mu_{1}, \mu_{2}\right]$ such that $\mu_{1} \leq \mu_{\text {inf }}<\mu_{2}$. Choose $\epsilon>0$, the tolerance level. If no knowledge of lower bounds of $\mu_{\text {inf }}$ is available, $\mu_{1}$ may be chosen as $\mu_{1}=\epsilon$.

(2) While $\left(\mu_{2}-\mu_{1}\right)>\epsilon$, set $\mu_{3}=\frac{\mu_{1}+\mu_{2}}{2}$, and check whether $\mu_{3}>\mu_{\text {inf }}$ by the above procedure. If $\mu_{3}>\mu_{\text {inf }}$, then set $\mu_{2}=\mu_{3}$, else set $\mu_{1}=\mu_{3}$.

(3) Output $\mu_{\text {inf }}=\frac{\mu_{1}+\mu_{2}}{2}$.

Unfortunately, the reachability problem for general hybrid systems is undecidable, see for example $[8,44]$. Therefore, the bisection method on $\mu$ that approximates the optimal value $\mu_{\text {inf }}$ cannot be guaranteed to terminate in a finite number of steps. However, in practice, the possibility of an endless loop can be averted by putting an a priori limit on the number of iterations, or by employing a grid based approximation as a termination condition. Nevertheless, to specify a subclass of hybrid systems, for which the above procedure can be guaranteed to terminate in finite steps, is still of great theoretical interest. We will focus on the decidability issue in the next section.

\section{Decidable switched linear systems}

In the previous section, we studied the robust performance analysis problem of linear hybrid systems. However, the termination of the proposed procedure in a finite number of steps is not guaranteed. The undecidability has been the main deterrent for the implementation of hybrid system theory to solve practical problems. Hence, a natural question is under what condition the procedure can terminate in a finite number of steps, i.e., decidable. To specify the decidable subclass of linear hybrid systems for the robust performance problems, two kinds of simplification may be employed. One way is to simplify the continuous variable dynamics of the hybrid systems, see for example [8]. It turns out that only a small portion of hybrid systems with simple or specific continuous variable dynamics, such as timed automata, multirate timed automata, rectangular hybrid automata and o-minimal hybrid systems, have finite state bisimular transition systems, and thus the reachability problem for such specific hybrid systems is known to be decidable [8]. To make the situation worse, it was shown in $[44,8]$ that even a minor generalization of the rectangular hybrid automata would quickly made the reachability problem undecidable. Although $[44,8]$ considered the continuous-time case only, the situation 
for the discrete-time case is similar. For the discrete-time case, it was shown in [33] that finite bisimulation could be obtained for discrete-time hybrid automata with nilpotent or controllable continuous variable LTI dynamics with single input. However, this approach may not be attractive to control applications, where simplified continuous variable dynamics may not be adequate to capture the system's dynamics. Alternatively, one may restrict the discrete event dynamics of the uncertain linear hybrid systems.

In this section, we will follow the second route and obtain a decidable subclass of hybrid systems, called switched linear systems, by simplifying the discrete event dynamics. In particular, for the switched linear systems, we do not consider partition of the state space, i.e., $\mathcal{P}_{q}=\mathcal{X}$ for all $q$. In other words, the transitions between any two modes may happen at any point in the state space. This gives us more freedom in the discrete event controller design, i.e., selecting the active mode $q$, without wondering about the implicit switching caused by the state space partition. It will be shown that this simplicity on the discrete dynamics induces the decidability of the performance analysis problem.

\section{I. Switched linear systems}

In this section, we consider a family of discrete-time perturbed linear systems described by the following difference equations.

$$
x(t+1)=A_{q} x(t)+B_{q} u(t)+E d(t), \quad t \in \mathbb{Z}^{+}
$$

where $x(t) \in \mathbb{R}^{n}$ is the state variable, $u(t) \in \mathcal{U}_{q} \subset \mathbb{R}^{m}$ is the continuous control input, and the disturbance input $d(t)$ is contained in $\mathcal{D} \subset \mathbb{R}^{r}$, the $l^{\infty}$ unit ball. The continuous variable dynamics of mode $q$ are defined by the state matrices $A_{q}, B_{q}$ and $E$ for every mode $q$. The finite set $Q=\left\{q_{1}, q_{2}, \ldots, q_{n}\right\}$ is called the set of modes. Notice that the main difference of switched systems from general hybrid systems (1) and (2) is that the activation region of each mode $\mathcal{P}_{q}$ is the whole state space, i.e., $\mathcal{P}_{q}=\mathcal{X}$ for all $q \in Q$ and $\mathcal{X}=\mathbb{R}^{n}$. This basically means that all the switchings are controllable and one has the freedom to switch to any mode in the whole state space. The simplification comes from the exclusion of implicit switching caused by the state space partition, also known as autonomous switching.

Combine the family of discrete-time linear systems (7) with a class of piecewise constant functions of time $\sigma: \mathbb{Z}^{+} \rightarrow Q$. Then we can define the following time-varying system as a discrete-time switched linear system

$$
x(t+1)=A_{\sigma(t)} x(t)+B_{\sigma(t)} u(t)+E d(t), \quad t \in \mathbb{Z}^{+} .
$$

The signal $\sigma(t)$ is called a switching signal. Let us denote the collection of all possible switching signals as $\Sigma_{a}$, which is usually called arbitrary switching signals in the literature [9].

Also consider the following output

$$
z(t)=C x(t)
$$

For this switched linear systems (8) and (9), we are also interested in determining a nonconservative bound for the $l^{\infty}$ induced norm from $d(t)$ to $z(t)$. It is known that the switched systems (8) and (9) has finite $l^{\infty}$ induced gain if and only if its undisturbed system is (semiglobally) asymptotically stable [25]. Therefore, we restrict our search only in the collection of asymptotically stabilizing control signals, $(\sigma(t), u(t))$, which is denoted as $\Sigma_{s} \times \mathcal{U}_{s}$. It is assumed that there exist asymptotically stabilizing control signals, $(\sigma(t), u(t))$, namely $\Sigma_{s} \times \mathcal{U}_{s}$ 
is nonempty. In the following, we will develop a procedure to determine $\mu_{\text {inf }}$ for the switched linear systems (8) and (9).

\section{2. $l^{\infty}$ Induced gain for switched linear systems}

We first introduce the definition of limit set, $\mathcal{L}^{(\sigma, u)}$, under given switching law $\sigma \in \Sigma_{a}$ and admissible control signal $u(t) \in \mathcal{U}_{\sigma(t)}$.

Definition 11. The limit set $\mathcal{L}^{(\sigma . u)}$ for the switched system (8), under given switching law $\sigma \in \Sigma_{a}$ and admissible control signal $u(t) \in \mathcal{U}_{\sigma(t)}$, is the set of states $x$ for which there exist an admissible sequence $d(t)$ and a non-decreasing time sequence $t_{k}$ (with $\lim _{k \rightarrow+\infty} t_{k}=+\infty$ ) such that

$$
\lim _{k \rightarrow+\infty} \Phi\left(0, t_{k}, \sigma(\cdot), u(\cdot), d(+)\right)=x
$$

where $\Phi\left(0, t_{k}, \sigma(\cdot), u(\cdot), d(\cdot)\right)$ denotes the value at the instant $t_{k}$ of the solution of (8) originating at $x_{0}=0$ and corresponding to $\sigma, u$ and $d$.

Intuitively, the limit set $\mathcal{L}^{(\sigma, u)}$ captures all the possible drifted states from the origin, $x_{0}=0$, via disturbances and parametric perturbations, while the switching sequence $\sigma(t)$ and control signal $u(t)$ are predetermined (just functions of time). For the asymptotically stabilizing control signals $(\sigma, u) \in \Sigma_{s} \times \mathcal{U}_{s}$, we know that the limit set $\mathcal{L}^{(\sigma \cdot u)}$ has the following property [45].

Lemma 1. For the asymptotically stabilizing control law $(\sigma, u)$, the limit set $\mathcal{L}^{(\sigma, u)}$ is nonempty and the state evolution of the switched system (8), for every initial condition $x(0)$ and admissible sequence $d(t) \in \mathcal{D}$, converges to $\mathcal{L}^{(\sigma, u)}$. Moreover, $\mathcal{L}^{(\sigma, u)}$ is bounded and if $x(t) \in \mathcal{L}^{(\sigma, u)}$ then $x(t+1)=A_{\sigma(t)} x(t)+B_{\sigma(t)} u(t)+E d(t) \in \mathcal{L}^{(\sigma, u)}$ for all possible $d(t) \in \mathcal{D}$.

Next, we define the limit set for the switched linear system (8) as

$$
\mathcal{L}=\inf \left\{\bigcap_{(\sigma, u) \in \Sigma_{s} \times \mathcal{U}_{s}} \mathcal{L}^{(\sigma, u)}\right\},
$$

where the intersection is with respect to any finite collection of the admissible control laws $(\sigma, u)$ that asymptotically stabilize the switched system (8). The "inf" is taken with respect to all these finite intersections of limit set $\mathcal{L}^{(\sigma, u)}$ which are polytopes with the origin in their interiors. The physical meaning of the limit set $\mathcal{L}$ can be described as follows. Assume that the state $x$ starts at the origin, which is the ideal case for disturbance attenuation. However, because of the disturbances and parametric perturbations, the state $x(t)$ will drift away from the origin no matter how one picks the mode $q(t)$ (switching sequence) or designs the control signal $u(t)$. The limit set $\mathcal{L}$ captures all such possible drifted states. In other words, the limit set $\mathcal{L}$ is the uncertainty of the system that has to be dealt with, and it represents a lower bound of the achievable performance level. Also, it can be shown that $\mathcal{L}$ has the property as follows.

Proposition 12. The set $\mathcal{L}$ is bounded and nonempty. For every initial condition $x(0)$, admissible $d(t) \in \mathcal{D}$, there exists an admissible control law $(\sigma, u)$ such that the state evolution of the switched system (8) converges to $\mathcal{L}$. In addition, $\mathcal{L}$ is controlled invariant for the switched system (8). 
Proof. The boundedness and non-emptiness of $\mathcal{L}$ comes from the fact that $\mathcal{L}^{(\sigma, u)}$ is nonempty and bounded for all asymptotically stabilizing control laws $(\sigma, u)$, and $0 \in \mathcal{L}^{(\sigma, u)}$. For any $x(0)$, there exists proper asymptotically stabilizing control laws $\left(\sigma_{1}, u_{1}\right)$ and finite $t_{1}$ such that $x(t) \in \mathcal{L}^{\left(\sigma_{1}, u_{1}\right)}$ for $t \geq t_{1}$ (from the definition of limit set $\left.\mathcal{L}^{\left(\sigma_{1}, u_{1}\right)}\right)$. If $x\left(t_{1}\right) \notin \mathcal{L}=\bigcap_{(\sigma, u)} \mathcal{L}^{(\sigma, u)}$, then there exists at least one limit set, say $\mathcal{L}^{\left(\sigma_{2}, u_{2}\right)}$, such that $x\left(t_{1}\right) \notin \mathcal{L}^{\left(\sigma_{2}, u_{2}\right)}$. From the definition of $\mathcal{L}^{\left(\sigma_{2} \cdot u_{2}\right)}$, we know that there exists finite $t_{2}>t_{1}$ such that $x(t) \in \mathcal{L}^{\left(\sigma_{2} \cdot u_{2}\right)}$ for $t \geq t_{2}$ under the control law $\left(\sigma_{2}, u_{2}\right)$. If $x\left(t_{2}\right) \notin \mathcal{L}$, then the arguments goes on until finally $x(t) \in \mathcal{L}$. We claim that with finite number of steps $x(t) \in \mathcal{L}$. This claim and invariance of the set $\mathcal{L}$ can be easily shown by contradiction.

It should be pointed out that the introduction of the limit set $\mathcal{L}^{(\sigma, u)}$ and $\mathcal{L}$ is for the purpose of proving the decidability of the procedures for $l^{1}$ analysis, namely the termination in a finite number of steps. It is not necessary to calculate these limit sets $\mathcal{L}^{(\sigma, u)}$ or $\mathcal{L}$ to implement the procedures for the determination of induced gains.

Define now the performance level $\mu$ set as

$$
\Omega_{\mu}=\left\{x:\|C x\|_{\infty} \leq \mu\right\} .
$$

Recursively define the sets $\mathcal{C}_{k}, k=0,1, \ldots$ as

$$
\mathcal{C}_{0}=\Omega_{\mu}, \quad \mathcal{C}_{k}=\operatorname{pre}\left(\mathcal{C}_{k-1}\right) \cap \mathcal{C}_{k-1}
$$

where the predecessor set for the switched system $(8)$, $\operatorname{pre}(\mathcal{C})$, is the set of states $x$ from which, despite disturbances, there exist a subsystem (switching signal $\sigma$ ) and continuous control signal $u$ driving the states to $\mathcal{C}$ in one step. The predecessor set for the switched system (8), pre $(\mathcal{C})$, can be calculated as

$$
\operatorname{pre}(\mathcal{C})=\bigcup_{q \in Q} \operatorname{pre}_{q}(\mathcal{C})
$$

where pre ${ }_{q}(\cdot)$ is defined in Section 3 with $\mathcal{P}_{q}=\mathbb{R}^{n}$.

By construction, $\mathcal{C}_{\infty}$ has the property that there exists a switching signal $\sigma(t)$ and admissible continuous control signal $u(t)$ with respect to which $\mathcal{C}_{\infty}$ is a positive controlled invariant for the switched system (8). Also it can be shown that $\mathcal{C}_{\infty}$ is the maximal controlled invariant subset contained in $\Omega_{\mu}$. Then, given $\mu>0$, there exists a switching signal $\sigma(t)$ and continuous control signal $u(t)$ such that the response of the switched system satisfies $\|z(t)\|_{l} \leq \mu$ for all $\|d(t)\|_{1 \infty} \leq 1$ if and only if the maximal controlled invariant subset contained in $\Omega_{\mu}, \mathcal{C}_{\infty}$, is nonempty and $0 \in \mathcal{C}_{\infty} \subseteq \Omega_{\mu}$.

We now give a proposition which guarantees that $\mathcal{C}_{\infty}$ can be finitely determined.'

Proposition 13. If $\mathcal{L} \subset$ int $\left\{\Omega_{\mu}\right\}$ for some $\mu>0$, then there exists $\mathbf{k}$ such that $\mathcal{C}_{\infty}=\mathcal{C}_{\mathbf{k}}$ and this $\mathbf{k}$ can be selected as the smallest integer such that $\mathcal{C}_{\mathbf{k}+1}=\mathcal{C}_{\mathbf{k}}$.

Proof. According to Proposition 12, there exists a finite integer $\mathbf{k}$ such that for all $x(0) \in \Omega_{\mu}$, $x(t) \in \mathcal{L} \subset \operatorname{int}\left\{\Omega_{\mu}\right\}(\forall t \geq \mathbf{k})$ for some proper control signals.

Also notice that the set $\mathcal{C}_{\mathrm{k}}$ has the property that for all $x \in \mathcal{C}_{\mathrm{k}}$, there always exist sequences of admissible control signals to make the trajectory starting from $x$ remain inside $\Omega_{\mu}$ for at least

${ }^{1}$ This represents an extension of similar results for linear time varying systems in [45] to switched systems. 
$\mathbf{k}$ steps, i.e., $x(t) \in \Omega_{\mu}, t=0,1, \ldots, \mathbf{k}$, for all possible $d(t) \in \mathcal{D}$. We will show that the above two facts imply $\mathcal{C}_{k+1}=\mathcal{C}_{k}$.

Proving by construction, it is assumed that $\mathcal{C}_{\mathrm{k}+1} \subset \mathcal{C}_{\mathbf{k}}$ (note that $\mathcal{C}_{\mathbf{k}+1} \subseteq \mathcal{C}_{\mathbf{k}}$ by definition). Hence, there exists $x \in \mathcal{C}_{\mathrm{k}}$ but $x \notin \mathcal{C}_{\mathrm{k}+1}$. Starting from this point $x$ (note that $x \in \mathcal{C}_{\mathrm{k}} \subseteq \Omega_{\mu}$ ), there exist sequences of $d \in \mathcal{D}$ such that $x(\mathbf{k}+1) \notin \Omega_{\mu}$ no matter how we choose control signals. This is because $x \notin \mathcal{C}_{\mathbf{k}+1}$ and the construction of $\mathcal{C}_{\mathbf{k}+1}$.

This leads to a contradiction. Therefore, $\mathcal{C}_{\mathbf{k}}=\mathcal{C}_{\mathbf{k}+1}$, and this implies that $\mathcal{C}_{\mathbf{k}}=\mathcal{C}_{\mathbf{k}+m}$, for $m \geq 0$. Thus $\mathcal{C}_{\infty}=\mathcal{C}_{\mathbf{k}}$.

The calculation of $\mu_{\mathrm{inf}}$ for switched systems (8) and (9) can now be solved by determining the maximal controlled invariant set $\mathcal{C}_{\infty}$ in $\Omega_{\mu}$ for several values of $\mu$ and checking whether or not it contains the origin. Note that in both cases we get an answer in a finite number of steps, although there is no a priori bound for such a number. In the first case, this is due to the above theorem. In the second case, this follows by the fact that the sequence of closed sets $\mathcal{C}_{\mathrm{k}}$ is ordered by inclusion and $\mathcal{C}_{\infty}$ is their intersection. Thus $0 \notin \mathcal{C}_{\infty}$ if and only if $0 \notin \mathcal{C}_{\mathbf{k}}$ for some $k$. These results suggest that the bisection algorithm (Algorithm 1) can be employed to approximate the optimal value $\mu_{\text {inf }}$ arbitrarily close.

\section{Robust optimal hybrid controller}

We have determined the minimal $l^{\infty}$ induced gain from $d(t)$ to $z(t)$ that can be achieved by linear hybrid/switched systems in the above two sections, thus answering the "robust performance analysis problem". Next, we turn to consider the "robust optimal control problem".

Our objective in this section is to design a hybrid control law, $c$, such that the closed-loop hybrid systems achieve the possible minimal $l^{\infty}$ induced gain from $d(t)$ to $z(t), \mu_{\text {inf. }}$. It has been shown in the previous section that the disturbance attenuation problem is solved if and only if the set $\Omega_{\mu}$ has a nonempty controlled invariant subset, $\mathcal{C}_{\infty}\left(\Omega_{\mu}\right)$. In addition, we know that the robust optimal control problem can be solved if and only if the closed loop trajectories remain in the maximal invariant subset of the performance level set $\mathcal{C}_{\infty}\left(\Omega_{\mu_{\text {inf }}}\right)$. In this section, we will present a systematic procedure for the hybrid controller design, which robustly drives the system, with proper initial conditions, to guarantee that the states remain within $\mathcal{C}_{\infty}\left(\Omega_{\mu_{\text {inf }}}\right)$ despite the disturbances. For notational simplicity, we denote the maximal controlled invariant subset $\mathcal{C}_{\infty}\left(\Omega_{\mu_{\text {inf }}}\right)$ as $\mathcal{C}$ in the following. Note that $\mathcal{C}$ is a (maybe non-convex) piecewise linear set.

A similar invariant control problem has been considered in $[46,47,42]$, where a receding horizon control approach was proposed to obtain the appropriate discrete modes and control signals by solving a collection of linear programming problems at each step. However, the computation burden is usually heavy for practical applications. Therefore, an explicit state feedback controller is desirable, especially for large dimensional systems or applications with fast dynamics.

In this section, we will design the hybrid control law in an explicit state feedback form, i.e., $\{q(x(t)), u(x(t))\}$. For such a purpose, we partition the region $\mathcal{C}$ into a finite number of convex subregions. First, we coarsely divide $\mathcal{C}$ into a finite union of convex piecewise linear sets $\mathcal{C}^{i}$, i.e., $\mathcal{C}=\bigcup_{i=1}^{m} \mathcal{C}^{i}$, which satisfy the property

$$
\operatorname{pre}_{q}\left(\bigcup_{i=1}^{m} \mathcal{C}^{i}\right)=\bigcup_{i=1}^{m} \operatorname{pre}_{\varphi}\left(\mathcal{C}^{i}\right) \text {, }
$$


for all feasible modes $q$. The existence and procedure to obtain such partition are described in the Appendix. It is assumed that the polytopic region $\mathcal{C}^{i}$ can be represented as

$$
\mathcal{C}^{i}=\left\{x: F^{i} x \leq g^{i}\right\},
$$

with proper dimensional matrix $F^{i}$ and vector $g^{i}$, for all $i=1,2, \ldots, m$.

Secondly, we refine the above polyhedral partition by subdividing each polyhedra $\mathcal{C}^{j}$ into

$$
\mathcal{C}_{q}^{i, j}=\operatorname{pre}_{q}\left(\mathcal{C}^{i}\right) \bigcap \mathcal{C}^{j}, \quad \text { for } i, j=1, \ldots, m, q \in Q .
$$

Note that $\mathcal{C}_{q}^{i, j}$ is a convex polyhedral set and it has the following property.

Proposition 14. A controlled invariant set $\mathcal{C}$ can be written as the following polytopic partition

$$
\mathcal{C}=\bigcup_{q \in Q} \bigcup_{i, j} \mathcal{C}_{q}^{i, j}
$$

where $\mathcal{C}_{q}^{i . j}$ is defined in (13).

Proof. First,

$$
\begin{aligned}
\bigcup_{i, j} \mathcal{C}_{q}^{i, j} & =\bigcup_{i, j}\left(\operatorname{pre}_{q}\left(\mathcal{C}^{i}\right) \bigcap \mathcal{C}^{j}\right)=\bigcup_{i}\left(\operatorname{pre}_{q}\left(\mathcal{C}^{i}\right)\right) \bigcap\left(\bigcup_{j} \mathcal{C}^{j}\right) \\
& =\operatorname{pre}_{q}\left(\bigcup_{i} \mathcal{C}^{i}\right) \bigcap \mathcal{C}=\operatorname{pre}_{q}(\mathcal{C}) \bigcap \mathcal{C} .
\end{aligned}
$$

Secondly, because pre ${ }_{q}(\mathcal{C}) \cap \mathcal{C} \subseteq \mathcal{C}$, for all $q \in Q$, so $\bigcup_{q \in Q}\left(\operatorname{pre}_{q}(\mathcal{C}) \cap \mathcal{C}\right) \subseteq \mathcal{C}$. On the other hand, $\mathcal{C}$ is controlled invariant, so $\mathcal{C} \subseteq \operatorname{pre}(\mathcal{C})=\bigcup_{q \in Q} \operatorname{pre}_{q}(\mathcal{C})$.

These polytopic subregion $\mathcal{C}_{q}^{i, j}$ have the following property. For all the states $x$ contained in the polytopic subregion $\mathcal{C}_{q}^{i, j}$, there exist admissible control signals $u \in \mathcal{U}_{q}$ such that drive $x$ into $\mathcal{C}^{i}$ (not $\mathcal{C}_{q}^{i, j}$ itself) along mode $q$ for all admissible disturbances. This property comes from the definition that $\mathcal{C}_{q}^{i, j}=\operatorname{pre}_{q}\left(\mathcal{C}^{i}\right) \cap \mathcal{C}^{j}$, so $x \in \mathcal{C}_{q}^{i, j}$ implies $x \in$ pre $_{q}\left(\mathcal{C}^{i}\right)$. The possible next step state $x^{\prime}$, which is guaranteed to be contained in $\mathcal{C}^{i}$, also falls into another polytopic subregion $\mathcal{C}_{q^{\prime}}^{i^{\prime}, i}$, for some $q^{\prime} \in Q$ and $i^{\prime} \in\{1,2, \ldots, m\}$. For the state $x^{\prime}$, there also exist control signals $u \in \mathcal{U}_{q^{\prime}}$ to drive $x^{\prime}$ into $\mathcal{C}^{i^{\prime}}$ along mode $q^{\prime}$. The procedure is repeated for the next step state, and so on. Therefore, the state trajectories under such control signals are contained in the region $\mathcal{C}=\bigcup_{i} \mathcal{C}^{i}$ despite disturbances. This observation suggests that one may pick the mode $q$ as the active mode for a polytopic subregion $\mathcal{C}_{q}^{i, j}$, and the existence of the admissible continuousvariable control signal $u \in \mathcal{U}_{q}$ is guaranteed, which makes the region $\mathcal{C}\left(=\bigcup_{q \in Q} \bigcup_{i, j} \mathcal{C}_{q}^{i . j}\right)$ robust controlled invariant. In the following, we will propose a systematic method to construct such continuous-variable control signals. In particular, a linear state feedback control law is designed for each polytopic subregion $\mathcal{C}_{q}^{i, j}$.

For such a purpose, an optimization problem for each vertex of the polytopic subregion $\mathcal{C}_{q}^{i, j}$ is formulated to calculate an admissible control signal for the vertex $x_{q}^{k} \in \operatorname{vert}\left\{\mathcal{C}_{q}^{i, j}\right\}$. Notice that the vertices can be easily determined by solving some linear programming problems once the polytopic region $\mathcal{C}_{q}^{i, j}$ is specified. The control signal for the vertex $x_{q}^{k}$ can be selected as the 
solution to the following minmax optimization problem:

$$
\begin{array}{r}
\min _{u \in \mathcal{U}_{q}} \max _{d \in \mathcal{D}}\left\|F^{j}\left[A_{q} x_{q}^{k}+B_{q} u+E d\right]\right\|_{\infty} \\
\text { s.t. }\left\{\begin{array}{l}
F^{i} B_{q} u \leq g^{i}-F^{i} A_{q} x_{q}^{k}-\delta_{q}^{i} \\
u \in \mathcal{U}_{q}
\end{array}\right.
\end{array}
$$

where $\delta_{q}^{i}=\max _{d \in \mathcal{D}}\left(F^{i} E d\right)$ componentwise, which incorporates the worst effects of the disturbance $d$. The optimal action of the controller is one that tries to minimize the maximum cost, and tries to counteract the worst disturbance and to keep the next step state inside the region $\mathcal{C}^{j}$ (not $\mathcal{C}_{q}^{i, j}$ itself). Of course, one may choose another cost functional, but the key point here is that the above constraints are linear inequalities in $u$ and nonempty, i.e., feasible. This is because $x_{q}^{k} \in \mathcal{C}_{q}^{i, j} \subset \operatorname{pre}_{q}\left(\mathcal{C}^{i}\right)$.

Because of the guaranteed feasibility of the above optimization problem for each vertex of the polytope $\mathcal{C}_{q}^{i, j}$, the admissible control signals for each vertex $x_{q}^{k}$ exist, which may be denoted as $u_{q}^{k}$. In the next step, we will construct the continuous variable control signals for the state contained in region $\mathcal{C}_{q}^{i, j}$ from the control signals at the vertices. The arguments are obtained through convexity.

Note that any $x \in \mathcal{C}_{q}^{i . j}$ can be (not uniquely) written as the convex combination of the vertices of $\mathcal{C}_{q}^{i, j}$, that is $x=\sum_{k} \alpha_{q}^{k}(x) x_{q}^{k}$, where the convex combination coefficients $\alpha_{q}^{k}(x) \geq 0$ and $\sum_{j} \alpha_{q}^{k}(x)=1$. We set the control signal $u(x)$ for state $x$ simply as the convex combination of the control signals at the vertex $u_{q}^{k}$. In particular,

$$
u(x)=\sum_{k} \alpha_{q}^{k}(x) u_{q}^{k}
$$

and $u(x) \in \mathcal{U}_{q}$ comes from the convexity of $\mathcal{U}_{q}$. And

$$
\begin{aligned}
F^{i}\left[A_{q} x+B_{q} u(x)\right] & =F^{i}\left[A_{q} \sum_{k} \alpha_{q}^{k}(x) x_{q}^{k}+B_{q} \sum_{k} \alpha_{q}^{k}(x) u_{q}^{k}\right] \\
& =\sum_{k} \alpha_{q}^{k}(x) F^{i}\left[A_{q} x_{q}^{k}+B_{q} u_{q}^{k}\right] \\
& \leq \sum_{k} \alpha_{q}^{k}(x)\left[g^{i}-\delta_{q}^{i}\right] \\
& =g^{i}-\delta_{q}^{i}
\end{aligned}
$$

holds. In other words, for any $x \in \mathcal{C}_{q}^{i . j}$, the control signal $u(x)$ given in (16) will drive the next state in $\mathcal{C}^{i}$ (not $\mathcal{C}_{q}^{i . j}$ itself) despite disturbances. Therefore, the control law of the form (16) solves the robust controlled invariance problem.

In summary, to make the performance level set $\mathcal{C}$ controlled invariant, the control law is given as follows. For $x(t) \in \mathcal{C}_{q}^{i . j}$, the discrete mode is selected as $q(t)=q$. This is always possible since

$$
x(t) \in \mathcal{C}_{q}^{i . j}=\operatorname{pre}_{q}\left(\mathcal{C}^{i}\right) \bigcap \mathcal{C}^{j} \subset \mathcal{P}_{q} .
$$

Secondly, the continuous variable control signal, $u(t)$, is of the form (16). In this expression, $\alpha_{q}^{k}(x)$ is the convex combination coefficients of $x(t)$ by the vertices of $\mathcal{C}_{q}^{i, j}$, and $u_{q}^{k}$ is the control 
signal for the corresponding vertices of $\mathcal{C}_{q}^{i, j}$. It has been shown that the vertex control signal $u_{q}^{k}$ can be derived by solving a linear programming problem, which can be solved off-line.

A control law of the above form (16) can be implemented as a piecewise linear state feedback controller. For example, let $X_{i, j}^{q}$ be a matrix whose columns are formed by the vertex vector of $\mathcal{C}_{q}^{i, j}$. The columns of matrix $U_{i, j}^{q}$ are the calculated continuous variable control vector, $u_{q}^{k}$, corresponding to each vertex of $\mathcal{C}_{q}^{i, j}$. A piecewise linear state feedback controller is then obtained by applying the control

$$
u(x)=\sum_{k} \alpha_{q}^{k}(x) u_{q}^{k}=U_{i, j}^{q}\left(X_{i, j}^{q^{T}} X_{i, j}^{q}\right)^{-1} X_{i, j}^{q^{T}} x
$$

where $(\cdot)^{T}$ stands for transpose, and $(\cdot)^{-1}$ inverse of a matrix. The convex combination coefficients $\alpha_{q}^{k}(x)$ can be calculated as $\left(X_{i, j}^{q^{T}} X_{i, j}^{q}\right)^{-1} X_{i, j}^{q^{T}} x$ if $\left(X_{i, j}^{q^{T}} X_{i, j}^{q}\right)$ is invertible. Otherwise another procedure is needed to generate the convex combination vector coefficients $\alpha_{q}^{k}(x)$. Note that all the calculations to derive the matrix $X_{i, j}^{q}$ and $U_{i, j}^{q}$ can be done off-line by linear programming techniques. The implementation of the control law only needs to calculate the convex combination coefficients vector $\alpha_{q}^{k}(x)$, which can be done by solving some linear equations. Therefore, this computational advantage makes the above method a good candidate to deal with high dimensional hybrid systems. This advantage also makes the method developed above distinct from the robust tracking and regulation controller design method presented in [46, $47,42]$, which is based on receding horizon control and on-line optimization.

Some remarks are in order. First, for some states $x(t) \in \mathcal{C}$, there may exist more than one feasible mode and admissible control signal. Then some criteria could be designed for the selection of $(q(x(t)), u(x(t)))$, e.g. the magnitude or energy of $u(x(t))$ etc. This flexibility may also lead to optimal control with respect to other kinds of cost functions. Secondly, the procedure developed here answers the robust optimal control problem in a decidable way even for the general hybrid systems (1) and (2) under the assumption that $\mathcal{C}$ is controlled invariant. In addition, although we only synthesize a hybrid control law to guarantee optimal $l^{\infty}$ induced gain $\mu_{\text {inf }}$ here, the procedure can be directly used to achieve any admissible disturbance attenuation level, $\mu>\mu_{\text {inf. }}$.

\section{Example}

Consider the following discrete-time linear hybrid system

$$
\begin{aligned}
& x(t+1)= \begin{cases}A_{0} x(t)+B_{0} u(t)+E_{0} d(t), & q=q_{0} \\
A_{1} x(t)+B_{1} u(t)+E_{1} d(t), & q=q_{1} .\end{cases} \\
& z(t)=C x(t)
\end{aligned}
$$

where

$$
\begin{aligned}
& A_{0}=\left[\begin{array}{ll}
1 & 1 \\
0 & 1
\end{array}\right], \quad B_{0}=\left[\begin{array}{l}
0 \\
1
\end{array}\right], \quad E_{0}=\left[\begin{array}{l}
0.1 \\
0.1
\end{array}\right] \\
& A_{1}=\left[\begin{array}{cc}
0 & 1 \\
-1 & -1
\end{array}\right], \quad B_{1}=\left[\begin{array}{l}
0 \\
1
\end{array}\right], \quad E_{1}=\left[\begin{array}{l}
0.1 \\
0.1
\end{array}\right], \quad C=\left[\begin{array}{ll}
1 & 1
\end{array}\right] .
\end{aligned}
$$

We assume that $u \in \mathcal{U}=[-1,1]$, and $d \in \mathcal{D}=[-1,1]$. 


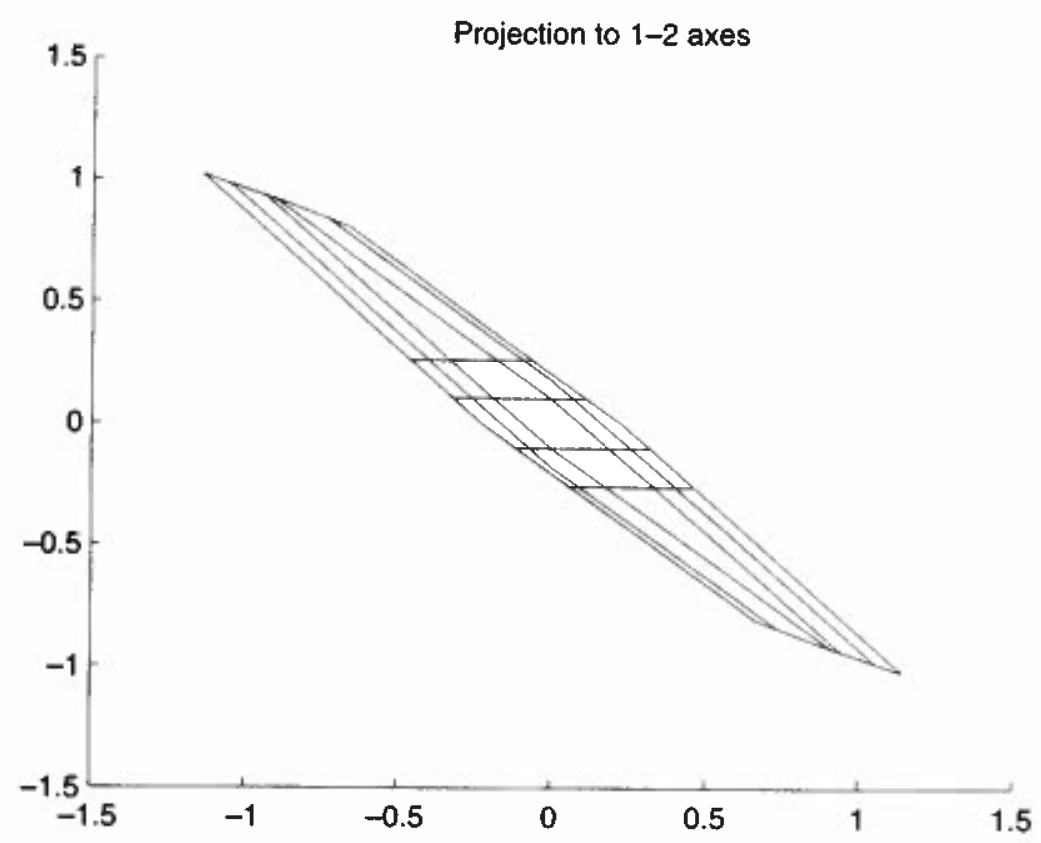

Fig. 1. The illustration of $\mathcal{C}_{\infty}\left(\Omega_{\mu_{\text {inf }}}\right)$.

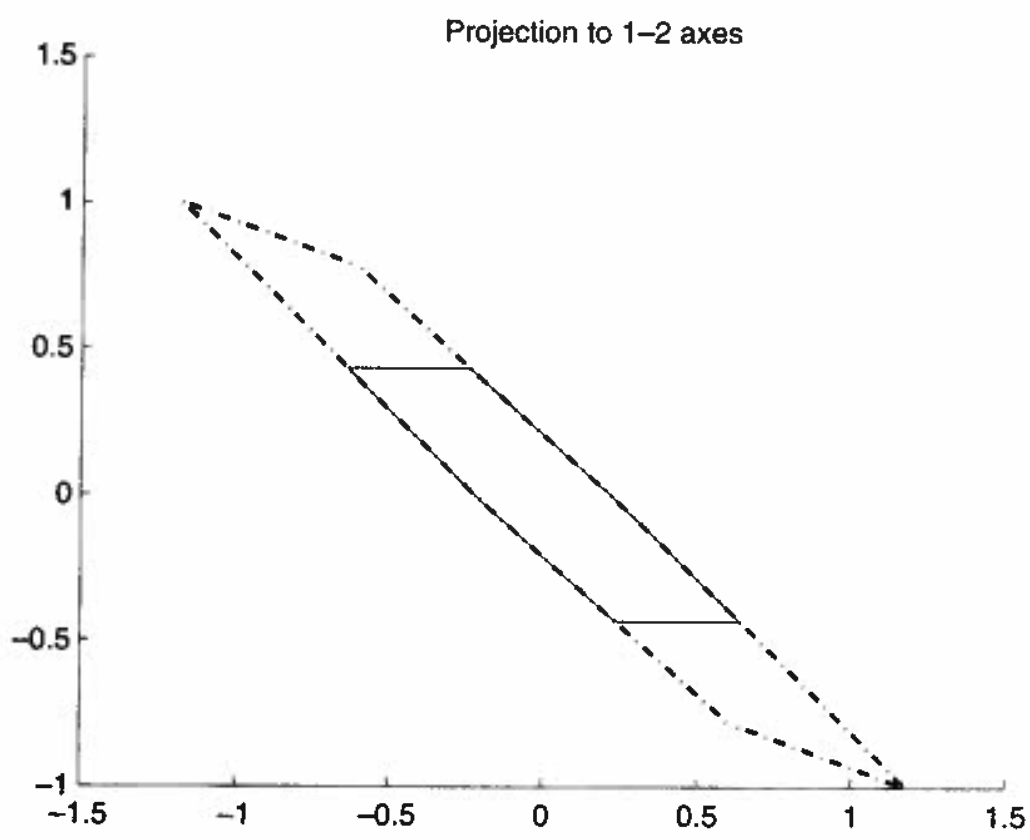

Fig. 2. The illustration of $\mathcal{C}_{q_{0}}^{1,1}$ (dashed line) and $\mathcal{C}_{q_{1}}^{1,1}$ (solid line).

First, determine the minimal $l^{\infty}$ induced gain from $d(t)$ to $z(t)$ that can be achieved by some admissible control law for the polytopic uncertain linear hybrid systems defined above. For such purpose, consider the region $\Omega_{\mu}=\{-\mu \leq|C x| \leq \mu\}$. Using Algorithm 1 (with error tolerance $\epsilon=0.01$ ) we compute the $\mu_{\mathrm{inf}}$, which is approximately $\mu_{\mathrm{inf}}=0.225$.

The maximal controlled invariant subset $\mathcal{C}_{\infty}\left(\Omega_{\mu_{\text {inf }}}\right)$ is calculated, as shown in Fig. 1.

Our next step is to design the invariant controller. Note that $\mathcal{C}$ in this example is a convex polyhedral set from calculation, i.e., $m=1$. Therefore, $\mathcal{C}_{q_{0}}^{1.1}$ can be calculated as $\mathcal{C}_{q_{0}}^{1.1}=$ pre $_{q_{0}}(\mathcal{C}) \cap \mathcal{C}$. Similarly, $\mathcal{C}_{q_{1}}^{1,1}=$ pre $_{q_{1}}(\mathcal{C}) \cap \mathcal{C}$ (see Fig. 2). 
Then we calculate the vertices control vectors for each single mode, by solving some linear programs. The coordinate of vertices and their corresponding control vector for $\mathcal{C}_{q_{0}}^{l .1}$ may be used to form the matrices $X_{q_{0}}$ and $U_{q_{0}}$ respectively. Similarly, we obtain the matrices $X_{q_{1}}$ and $U_{q_{1}}$ from $\mathcal{C}_{q_{1}}^{1, \mathrm{I}}$. For example, the coordinate of vertices and their corresponding control vector for $\mathcal{C}_{q_{1}}^{1,1}$ is represented as the matrices $X_{q_{1}}$ and $U_{q_{1}}$ as follows.

$$
X_{q_{1}}^{T}=\left(\begin{array}{cc}
-0.2200 & 0.0000 \\
0.2200 & 0.0000 \\
-0.6355 & 0.4328 \\
0.6355 & -0.4328 \\
-0.2350 & 0.4328 \\
0.2350 & -0.4328
\end{array}\right), \quad U_{q_{1}}^{T}=\left(\begin{array}{c}
-0.2200 \\
0.2200 \\
-0.5820 \\
0.5820 \\
-0.1815 \\
0.1815
\end{array}\right)
$$

For any state $x(t) \in \mathcal{C}_{\infty}\left(\Omega_{\mu_{\text {inf }}}\right)$, the hybrid control law is designed as the following:

- If $x \in \mathcal{C}_{q_{0}}^{1,1}$, then the $u$ is given by Eq. (17) with $X_{q}=X_{q_{0}}$ and $U_{q}=U_{q_{0}}$. And the discrete mode is selected as $q(t)=q_{0}$.

- If $x \notin \mathcal{C}_{q_{0}, 1}^{1,1}$, then $x$ must be contained in $\mathcal{C}_{q_{1}}^{1,1}$ (because $\mathcal{C}_{q_{1}}^{1,1} \cup \mathcal{C}_{q_{1}}^{1,1}=\mathcal{C}_{\infty}\left(\Omega_{\mu_{\text {inf }}}\right)$ ). In this case, the $u$ is given by Eq. (17) with $X_{q}=X_{q_{1}}$ and $U_{q}=U_{q_{1}}$. And the discrete mode is selected as $q(t)=q_{1}$.

\section{Concluding remarks}

In this paper, we studied the robust performance analysis and synthesis problems of classes of linear hybrid systems in the framework of invariant set theory. The robust performance problems were equivalently transformed into the controlled robust invariance problems for a specific region decided by the performance level. Based on the geometric condition for robust controlled invariance, a bisection-based procedure was proposed to determine the optimal disturbance attenuation level $\mu_{\text {inf. The decidability issue of the robust performance analysis problem was }}$ discussed, and switched linear systems were specified as a decidable subclass of the linear hybrid systems. The decidability comes from the simplicity of the discrete event dynamics. It is worth pointing out that this specific simple subclass of hybrid systems still can model a large class of practical systems, such as multi-controller supervisory control systems, controller failures, fault diagnosis and control reconfiguration etc. For example, in [47,48], a class of networked control systems with uncertain delays and package dropout effects was modeled as such switched systems. Finally, a systematic procedure for explicit hybrid robust optimal controller design was given, which was based on polyhedral algebra and linear programming techniques. The robust optimal controller is in the form of a piecewise linear state feedback control law. It is interesting to note that in [17] a variety of optimal control problems of piecewise linear systems could also be solved by piecewise linear state feedback control laws, which were obtained through multiparametric linear (or quadratic) programming. However, different approaches were employed in this paper. Based on predecessor operator and polyhedral algebra, we directly partitioned the performance level set into conic subsets, and designed linear feedback gain for each subset.

\section{Appendix}

Notation. Given two sets $Y \subset \mathbb{R}^{n}$ and $Z \subset \mathbb{R}^{n}$, the complement of $Y$ is $Y^{c}=\left\{y \in \mathbb{R}^{n}: y \notin Y\right\}$, the Minkovski sum $Y \oplus Z=\{y+z: y \in Y, z \in Z\}$ and the Pontryagin difference $Y \sim Z=\left\{x \in R^{n}: x+z \in Y, \forall z \in Z\right\}$. 
First, we present some primary results on the Minkovski sum and Pontryagin difference.

Lemma 2. Given two sets $X \subset \mathbb{R}^{n}$ and $Y \subset \mathbb{R}^{n}$, we have

$$
(X \sim Y) \oplus Y \subseteq X \subseteq(X \oplus Y) \sim Y .
$$

Proof. First, for any $z \in(X \sim Y), z+y \in X$ for all $y \in Y$, so $(X \sim Y) \oplus Y \subseteq X$. Secondly, for any $x \in X, x+y \in X \oplus Y$ for any $y \in Y$, so $x \in(X \oplus Y) \sim Y$ by definition. Therefore, $X \subseteq(X \oplus Y) \sim Y$.

Lemma 3 ([3I]). If $X$ is a (non-convex) piecewise linear set and $Y$ is a polytope, then the Pontryagin difference $X \sim Y$ can be written as a finite union of convex polyhedral sets, i.e., $X \sim Y=\bigcup_{i} \phi_{i}$.

Lemma 4. Assume that $X \sim Y=\bigcup_{i} \phi_{i}$, where $X$ is a piecewise linear set, $Y$ is a polytope and $\phi_{i}$ is a convex polyhedral set. Then

$$
X=\bigcup_{i}\left(\phi_{i} \oplus Y\right)
$$

Proof. First, $X \supseteq(X \sim Y) \oplus Y=\left(\bigcup_{i} \phi_{i}\right) \oplus Y=\bigcup_{i}\left(\phi_{i} \oplus Y\right)$. On the other hand, $\bigcup_{i}\left(\phi_{i} \oplus Y\right) \sim Y=\left[\bigcup_{i}\left(\phi_{i}\right) \oplus Y\right] \sim Y \supseteq \bigcup_{i} \phi_{i}=X \sim Y$, which implies $\bigcup_{i}\left(\phi_{i} \oplus Y\right) \supseteq X$. Therefore, $X=\bigcup_{i}\left(\phi_{i} \oplus Y\right)$.

Note that $\phi_{i} \oplus Y$ is a convex polyhedral set. Therefore, we write the non-convex piecewise linear set $X$ into a finite union of convex polyhedral sets, i.e., $X=\bigcup_{i}\left(\phi_{i} \oplus Y\right)$. And it has the following property.

\section{Lemma 5.}

$$
X \sim Y=\bigcup_{i}\left[\left(\phi_{i} \oplus Y\right) \sim Y\right]
$$

Proof. $X \sim Y=\bigcup_{i}\left(\phi_{i} \oplus Y\right) \sim Y \supseteq \bigcup_{i}\left[\left(\phi_{i} \oplus Y\right) \sim Y\right] \supseteq \bigcup_{i} \phi_{i}=X \sim Y$. Therefore, $X \sim Y=\bigcup_{i}\left[\left(\phi_{i} \oplus Y\right) \sim Y\right]$.

Based on the above properties, we will show that the one-step robust predecessor set for a non-convex piecewise linear set $\Omega$ can be written as a finite union of one-step robust predecessor set of its subsets. Consider the operation pre $_{q}(\cdot)$

$$
\begin{aligned}
\operatorname{pre}_{q}(\Omega) & =\left\{x \in \mathcal{P}_{q} \mid \exists u \in \mathcal{U}_{q}: A_{q} x+B_{q} u+E d \in \Omega, \forall d \in \mathcal{D}\right\} \\
& =\left\{x \in \mathcal{P}_{q} \mid \exists u \in \mathcal{U}_{q}: A_{q} x+B_{q} u \in \Omega \sim E \mathcal{D}\right\} \\
& =\operatorname{Proj}_{x}\left\{\left[\begin{array}{l}
x \\
u
\end{array}\right] \in \mathcal{P}_{q} \times \mathcal{U}_{q} \mid\left[A_{q}, B_{q}\right]\left[\begin{array}{l}
x \\
u
\end{array}\right] \in \Omega \sim E \mathcal{D}\right\}
\end{aligned}
$$

where $\operatorname{Proj}_{x}$ is the projection operator, which can be calculated by the Fourier-Motzkin elimination method. Note that $(\Omega \sim E \mathcal{D}$ ) can be written as a finite union of convex polyhedral sets $\phi_{i}$, that is $\Omega \sim E \mathcal{D}=\cup_{i} \phi_{i}$.

Because of Lemma 4 , the region $\Omega$ can be divided into a finite union of convex sets as:

$$
\Omega=\bigcup_{i}\left(\phi_{i} \oplus E \mathcal{D}\right)
$$


And it has the property according to Lemma 5 that

$$
\Omega \sim E \mathcal{D}=\left[\bigcup_{i}\left(\phi_{i} \oplus E \mathcal{D}\right)\right] \sim E \mathcal{D}=\bigcup_{i}\left[\left(\phi_{i} \oplus E \mathcal{D}\right) \sim E \mathcal{D}\right]
$$

Therefore,

$$
\begin{aligned}
\operatorname{pre}_{q}(\Omega) & =\operatorname{Proj}_{x}\left\{\left[\begin{array}{l}
x \\
u
\end{array}\right] \in \mathcal{P}_{q} \times \mathcal{U}_{q} \mid\left[A_{q}, B_{q}\right]\left[\begin{array}{l}
x \\
u
\end{array}\right] \in \Omega \sim E \mathcal{D}\right\} \\
& =\operatorname{Proj}_{x}\left\{\left[\begin{array}{l}
x \\
u
\end{array}\right] \in \mathcal{P}_{q} \times \mathcal{U}_{q} \mid\left[A_{q}, B_{q}\right]\left[\begin{array}{l}
x \\
u
\end{array}\right] \in \bigcup_{i}\left[\left(\phi_{i} \oplus E \mathcal{D}\right) \sim E \mathcal{D}\right]\right\} \\
& =\bigcup_{i} \operatorname{Proj}_{x}\left\{\left[\begin{array}{l}
x \\
u
\end{array}\right] \in \mathcal{P}_{q} \times \mathcal{U}_{q} \mid\left[A_{q}, B_{q}\right]\left[\begin{array}{l}
x \\
u
\end{array}\right] \in\left(\phi_{i} \oplus E \mathcal{D}\right) \sim E \mathcal{D}\right\} \\
& =\bigcup \operatorname{pre}_{q}\left(\phi_{i} \oplus E \mathcal{D}\right) .
\end{aligned}
$$

Denote $\phi_{i} \oplus E \mathcal{D}$ as $\Phi_{i}$, then $\Omega=\bigcup_{i} \Phi_{i}$ and

$$
\operatorname{pre}_{q}\left(\bigcup_{i} \Phi_{i}\right)=\bigcup_{i} \operatorname{pre}_{q}\left(\Phi_{i}\right)
$$

In conclusion, given a piecewise linear region $\Omega$, which may be non-convex, it is always possible to partition the region $\Omega$ into a finite number of polyhedrons $P_{i}$, such that pre ${ }_{q}(\Omega)=$ $\bigcup_{i}$ pre $_{q}\left(P_{i}\right)$ for all $q$, and $\Omega=\bigcup_{i} P_{i}$. In addition, the proof of this claim also gives a method to calculate such a partition by using the Minkovski sum and Pontryagin difference.

\section{References}

[1] P. Antsaklis (Ed.), Proceedings of the IEEE: Special Issue on Hybrid Systems, vol. 88, IEEE Press, 2000.

[2] A.V. Schaft, H. Schumacher. An Introduction to Hybrid Dynamical Systems, in: Lecture Notes in Control and Information Sciences, vol, 251, Springer-Verlag. London, 2000.

[3] E. Sontag, Interconnected automata and linear systems: A theoretical framework in discrete-time, in: T.A. Henzinger, R. Alur, D. Sontag (Eds.), Hybrid Systems III, in: Lecture Notes in Computer Science, vol, 1066, Springer-Verlag, 1996, pp. 436-448.

[4] R. Alur, C. Coufcoubetis, N. Halbwachs, T. Henzinger, P.H. Ho, X. Nicollin, A. Oliveiro, J. Sifakis, S. Yovine, The algorithmic analysis of hybrid systems, Theoret. Comput. Sci. 138 (1995) 3-34.

[5] A. Bemporad, M. Morari, Control of systems integrating logic, dynamics, and constraints, Automatica 35 (3) (1999) $407-427$.

[6] J. Aubin, J. Lygeros, M. Quincampoix, S. Sastry. N. Seube, Impulse differential inclusions: A viability approach to hybrid systems, IEEE Trans. Automat. Control 47 (1) (2002) 2-20.

[7] E. Asarin, O. Bournez, T. Dang, O. Maler, A. Pnueli, Effective synthesis of switching controllers for linear systems, in: P.J. Antsaklis (Ed.), Proceedings of the IEEE: Special Issue on Hybrid Systems, vol. 88, IEEE Press, 2000, pp. $1011-1025$.

[8] R. Alur, T. Henzinger, G. Lafferriere, G.J. Pappas, Discrete abstractions of hybrid systems, in: P.J. Antsaklis (Ed.). Proceedings of the IEEE: Special Issue on Hybrid Systems, vol, 88, IEEE Press, 2000, pp. 971-984.

[9] D. Liberzon, A.S. Morse, Basic problems in stability and design of switched systerns, IEEE Control. Syst. Mag. 19 (5) (1999) 59-70.

[10] R.A. Decarlo, M.S. Branicky, S. Pettersson, B. Lennartson. Perspectives and results on the stability and stabilizability of hybrid systems, in: P.J. Antsaklis (Ed.), Proceedings of the iEEE: Special Issue on Hybrid Systems. vol. 88, IEEE Press. 2000, pp. 1069-1082.

[11] M. Johansson, Piecewise linear control systems, Ph.D. Thesis, Lund Institute of Technology, Sweden, 1999. 
[12] J.P. Hespanha, Uniform stability of switched linear systems: Extensions of lasalle's invariance principle, IEEE Trans. Automat. Control. 49 (4) (2004) 470-482.

[13] D. Liberzon, Switching in Systems and Control, Birkhauser, Boston, 2003.

[14] Z. Sun, S.S. Ge, Analysis and synthesis of switched linear controt systems, Automatica 41 (2) (2005) 181-195.

[15] A. Bemporad, G. Ferrari-Trecate, M. Morari, Observability and controllability of piecewise affine and hybrid Systems, IEEE Trans. Automat. Control. 45 (10) (2000) 1864-1876.

[16] Z. Sun, S.S. Ge, Switched Lineat Systems: Contro] and Design, Springer-Ver]ag. 2005.

[17] A. Bemporad, F. Borrelli, M. Morari, On the optimal control law for linear discrete time hybrid systems. in: Hybrid Systerns: Computation and Control, in: Lecture Notes in Computer Science, vol. 2289, Springer, 2002, pp. 105-119.

[18] X. Xu, P.J. Antsaklis, Optimal control of switched systems based on parameterization of the switching instants. IEEE Trans. Automat. Control. 49 (1) (2004) 2-16.

[19] G. Zhai, B. Hu, K. Yasuda, A.N. Michel, Disturbance attenuation properties of time-controlted switched systems. J. Franklin lnst. 338 (2001) 765-779.

[20] G. Zhai, B. Hu, K. Yasuda, A.N. Michel, Qualitative analysis of discrete-time switched systems, in: Proc. 2002 American Contr. Conf., vol. 3, 2002, pp. 1880-1885.

[21] J.P. Hespanha, Root-mean-square gains of switched linear systems, IEEE Trans. Automat. Control 48 (11) (2003) 2040-2045.

[22] A.V. Savkin. R.J. Evans, A new approach to robust control of hybrid systems over infinite time, IEEE Trans. Automat. Control. 43 (9) (1998) 1292-1296.

[23] G. Ferrari-Trecate, F. Cuzzola, D. Mignone, M. Morari, Analysis and control with performance of piecewise affine and hybrid systems, in: Proc. 2001 American Control Conf., 2001, pp. 200-205.

[24] M.A. Dahleh, I. Diaz-Bobillo, Control of Uncertain Systems: A Linear Programming Approach. Prentice Hall. 1994.

[25] H. Lin, P.J. Antsaklis, Disturbance attenuation properties for discrete-time uncertain switched linear systems, in: Proc. 42nd IEEE Conf. Decision Control, 2003, pp. 5289-5294.

[26] H. Lin, P. Antsaklis, Uniformly ultimate boundedness control for uncertain switched linear systems, ISIS Technical Report, ISIS-2003-004, Univ. of Notre Dame, August 2003.

[27] H. Lin, P. Antsaklis, Disturbance altenuation in classes of uncertain linear hybrid systems, in: Proc. 2004 American Contr. Conf., 2004, pp. 566-571.

[28] D.P. Bertsekas, Infinite-time reachability of state-space regions by using feedback control, IEEE Trans. Automat. Control. 17 (5) (1972) 604-613.

[29] F. Blanchini, Ultimate boundedness control for uncertain discrete-time systems via set-induced Lyapunov functions, IEEE Trans. Automat. Control. 39 (2) (1994) 428-433.

[30] C.E.T. Dórea, J.C. Hennet, On $(a, b)$-invariance of polyhedral domains for discrete-time systems, Proc. 35th IEEE Conf. Decision Control, 1996, pp. 4319-4324.

[31] E. Kerrigan, Robust constraint satisfaction: Invariant sets and predictive control, Ph.D. Thesis, University of Cambridge, UK, 2000.

[32] F. Blanchini, Set invariance in control, Automatica 35 (11) (1999) 1747-1767.

[33] R. Vidal, S. Schaffert, O. Shakernia, J. Lygeros, S. Sastry, Decidable and semi-decidable controller synthesis for classes of discrete time hybrid systems, in: Proceedings of the 40th IEEE Conference on Decision and Control. 2001 , pp. 1243-1248.

[34] M. Jirstrand, Invariant sets for a class of hybrid systems, in: Proc. 37th IEEE Conf. Decision Control. 1998, pp. 3699-3704.

[35] L. Berardi, E.D. Santis, M.D.D. Benedetto, G. Pola. Approximations of maximal controlled safe sets for hybrid systems, in: Nonlinear and Hybrid Systems in Automotive Control, Springer, 2003, pp. 335-349.

[36] J. Lygeros, C. Tomlin, S. Sastry, Controllers for reachability specifications for hybrid systems, Automatica 35 (3) (1999) 349-370.

[37] X.D. Koutsoukos, Analysis and design of piecewise linear hybrid dynamical systems, Ph.D. Thesis, University of Notre Dame, Notre Dame, IN, USA, 2000.

[38] A. Puri, P. Varaiya, Verification of hybrid systems using abstractions, in: Hybrid Systems II, in: Lecture Notes in Computer Science, vol. 999, Springer, 1995, pp. 359-369.

[39] J. Stiver, X.D. Koutsoukos, P. Antsaklis, M. Lemmon, An invariant based approach to the design of hybrid control systems, Internat. J. Robust Nonlinear Control 11 (5) (2001) 453-478.

[40] P. Caines, Y. Wei. Hierarchical hybrid control systems: A lattice theoretic formulation. IEEE Trans. Automat. Control 43 (4) (1998) 501-508. 
[41] M. Broucke, A geometric approach to bisimulation and verification of hybrid systems, in: Proceedings of the 37th Conference on Decision and Control, vol. 4, 1998. pp. 4277-4282.

[42] H. Lin. P. Antsaklis, Robust tracking and regulation control of uncertain piecewise linear systems, ISIS Technical Report, ISIS-2003-005, Univ. of Notre Dame, October 2003.

[43] T. Motzkin (Ed.), The Theory of Linear Inequalities, Rand Corp., Santa Monica, CA, 1952.

[44] T. Henzinger, P.W. Kopke, A. Puri, P. Varaiya, What's decidable about hybrid automata? J. Comput. System Sci. 57 (1998) 94-124.

[45] F. Blanchini, S. Miani, M. Sznaier, Robust performance with fixed and worst case signals for uncertain time-varying systems, Automatica 33 (12) (1997) 2183-2189.

[46] H. Lin, P. Antsaklis, Controller synthesis for a class of uncertain piecewise linear hybrid dynamical systems, in: Proc. 4lst IEEE Conf. Decision Control, 2002, pp. 3188-3193.

[47] H. Lin, P. Antsaklis, Robust regulation of polytopic uncertain linear hybrid systems with networked control system applications, in: D. Liu, P.J. Antsaklis (Eds.), Stability and Control of Dynamical Systems with Applications: A Tribute to Anthony N. Michel, Birkhauser, 2003, pp. 71-96.

[48] H. Lin, G. Zhai, P.J. Antsaklis, Robust stability and disturbance attenuation analysis of a class of networked control systems, in: Proc. 42nd IEEE Conf. Decision Control, 2003, pp. 1182-1187. 